\title{
RNA-seq analysis reveals considerable genetic diversity and provides genetic markers saturating all chromosomes in the diploid wild wheat relative Aegilops umbellulata
}

\author{
Moeko Okada', Kentaro Yoshida ${ }^{1 *}$ D, Ryo Nishijima', Asami Michikawa', Yuka Motoi ${ }^{2}$, Kazuhiro Sato \\ and Shigeo Takumi ${ }^{1}$
}

\begin{abstract}
Background: Aegilops umbellulata Zhuk. $(2 n=14)$, a wild diploid wheat relative, has been the source of trait improvement in wheat breeding. Intraspecific genetic variation of Ae. umbellulata, however, has not been well studied and the genomic information in this species is limited.

Results: To develop novel genetic markers distributed over all chromosomes of Ae. umbellulata and to evaluate its genetic diversity, we performed RNA sequencing of 12 representative accessions and reconstructed transcripts by de novo assembly of reads for each accession. A large number of single nucleotide polymorphisms (SNPs) and insertions/deletions (indels) were obtained and anchored to the pseudomolecules of Ae. tauschii and barley (Hordeum vulgare L.), which were regarded as virtual chromosomes of Ae. umbellulata. Interestingly, genetic diversity in Ae. umbellulata was higher than in Ae. tauschii, despite the narrow habitat of Ae. umbellulata. Comparative analyses of nucleotide polymorphisms between Ae. umbellulata and Ae. tauschii revealed no clear lineage differentiation and existence of alleles with rarer frequencies predominantly in Ae. umbellulata, with patterns clearly distinct from those in Ae. tauschii.

Conclusions: The anchored SNPs, covering all chromosomes, provide sufficient genetic markers between Ae. umbellulata accessions. The alleles with rarer frequencies might be the main source of the high genetic diversity in Ae. umbellulata.
\end{abstract}

Keywords: Aegilops umbellulata, Aegilops tauschii, Barley, DNA markers, RNA sequencing, Synteny

\section{Background}

Aegilops umbellulata Zhuk. $(2 \mathrm{n}=14)$, a wild diploid wheat relative, is distributed in West Asia and is known as the U-genome donor of Ae. columnaris and Ae. triaristata [1, 2]. Ae. umbellulata (UU genome) has crossability with tetraploid wheat ( $T$. turgidum L.; AABB genome), which allows generation of synthetic hexaploids (AABBUU genome) through ABU triploids. Some

\footnotetext{
* Correspondence: kentaro.yoshida@port.kobe-u.ac.jp

${ }^{1}$ Graduate School of Agricultural Science, Kobe University, Rokkodai 1-1,

Nada-ku, Kobe 657-8501, Japan

Full list of author information is available at the end of the article
}

combinations of interspecific crosses between Ae. umbellulata accessions and tetraploid wheat result in hybrid incompatibility, such as severe growth abortion and grass-clump dwarfness [3]. This observation suggests the existence of unrevealed genetic polymorphisms in Ae. umbellulata that potentially vary phenotypic traits.

Ae. umbellulata have been used for breeding of bread wheat and is a considerable resource of disease resistance genes [4-10]. Leaf rust and stripe rust resistance genes $[6,8,11]$ and high-molecular weight glutenin subunits $[5,12]$ have been introduced into bread wheat cultivars. Chhuneja et al. (2008) [6] and Bansal et al. (2017)

(C) The Author(s). 2018 Open Access This article is distributed under the terms of the Creative Commons Attribution 4.0 International License (http://creativecommons.org/licenses/by/4.0/), which permits unrestricted use, distribution, and 
[8] established introgression lines of leaf and stripe rust resistance genes derived from synthetic hexaploids (AABBUU). The cross of the synthetic hexaploids (AABBUU) with $T$. aestivum cv. Chinese Spring $P h^{I}$, which carries an epistatic inhibitor of $P h 1$ gene, induced homologous pairing and resulted in transfer of the leaf and stripe rust resistance genes of Ae. umbellulata into the bread wheat $T$. aestivum. Although Ae. umbellulata provides valuable genetic resources for breeding of bread wheat, it has not been well studied and information on its genome is limited. Evaluation of intraspecific genetic diversity based on genome-wide polymorphisms in Ae. umbellulata would impart practical information for designing genetic markers, facilitating the efficient use of Ae. umbellulata for breeding.

Since the tribe Triticeae has a large genome, most of which is occupied by repetitive sequences, development of high-quality physical maps and whole genome sequencing are challenging. RNA sequencing (RNA-seq) is one of the solutions for detection of single nucleotide polymorphisms (SNPs) and evaluation of genetic diversity by avoiding these genome complexities of the Triticeae. RNA-seq approaches for identifying novel genetic markers have been applied to several Triticeae species such as $T$. monococcum [13] and Ae tauschii [14-16]. RNA-seq has the advantage of direct detection of SNPs linked to causal genes for targeted phenotypes. RNA-seq-based bulked segregant analysis narrowed down the genome location of a wheat yellow rust resistance gene, $\mathrm{Yr} 15$, and a wheat spot blotch resistance gene, Sb3, within $0.77 \mathrm{cM}$ and $0.15 \mathrm{cM}$ intervals, respectively $[17,18]$.

Recently, the highest-quality genome sequences have been developed in the diploid Triticeae species barley (Hordeum vulgare L.) $[19,20]$ and Ae. tauschii $[21,22]$. By utilizing highly conserved chromosomal synteny across Triticeae species [23, 24], the pseudomolecules of barley and Ae. tauschii can be regarded as virtual chromosomes of other Triticeae species. By combining RNA-seq with positional information from this synteny, a large number of SNPs and indels can be anchored to the chromosomes, facilitating design of genome-wide genetic markers [16]. The RNA-seq-based approach for marker development is considered applicable to other wild wheat species when enough genomic information is lacking.

Here, to evaluate genetic polymorphisms and capture genetic markers in Ae. umbellulata, transcripts of 12 representative accessions of Ae. umbellulata were first reconstructed by de novo assembly of reads from RNA-seq on the Illumina MiSeq platform. Using the deduced transcript sequences, a large number of SNPs and indels between the Ae. umbellulata accessions were detected and anchored to the barley and Ae. tauschii pseudomolecules. Comparative analysis of DNA polymorphisms between Ae. umbellulata and Ae. tauschii revealed relatively high genetic diversity in Ae. umbellulata.

\section{Methods}

Plant materials, library construction and RNA sequencing

Twelve accessions of Ae. umbellulata were chosen from the wheat genetic resources database of the National BioResource Project-Wheat (Japan, https://shigen.ni g.ac.jp/wheat/komugi/top/top.jsp) to represent the diversity of this species (Fig. 1; Table 1). T. urartu KU-199-5 was used as the outgroup species for the comparative analysis between Ae. umbellulata and Ae. tauschii. Total RNA was extracted from leaves of Ae. umbellulata and T. urartu at the seedling stage using a Sepasol-RNA I Super G solution (Nacalai Tesque, Kyoto, Japan). The total RNA was treated with DNase I at $37{ }^{\circ} \mathrm{C}$ for $20 \mathrm{~min}$ to remove contaminating DNA. A total of 6 to $10 \mu \mathrm{g}$ of RNA was used for constructing paired-end libraries. The libraries were constructed with TruSeq RNA Library Preparation Kit v2 (Illumina, San Diego, CA, USA) according to the manufacturer's instructions, and were sequenced with 300-bp paired-end reads on an Illumina MiSeq sequencer.

\section{De novo assembly of reads from RNA-seq}

Low-quality bases (average quality score per $4 \mathrm{bp}<30$ ), adapter sequences, and reads $<100 \mathrm{bp}$ were removed using the Trimmomatic version 0.33 tool [25]. The paired-end reads were assembled with Trinity version 2.0.6 software to reconstruct transcripts for each accession [26, 27]. If a gene had multiple isoforms, the first transcript sequence designated by Trinity was chosen as a unigene. A set of unigenes was made for each accession according to our previous report [16], and was used as a reference transcript dataset. Paired-end reads from each accession were aligned to the reference transcripts using the Bowtie 2 [28]. SAMtools and Coval software were used for SNP and indel calling [29, 30]. SNPs and indels were called when over $95 \%$ of the aligned sequences were different from those of the reference transcripts at positions with read depth $>10$. Sequence data have been deposited to DDBJ Sequence Read Archive DRA006404.

\section{Mapping the assembled transcripts, SNPs and indels to barley and Ae. tauschii genome sequences}

The transcripts were mapped to the barley (Hordeum vulgare L.) reference genome "ASM32608v1 masked" [19] from the Ensembl Plants database [31] and to the Ae. tauschii genome "PRJNA341983" from the NCBI database [21] using Gmap software version 2014-12-31 [32] and bedtools [33]. Based on the transcripts mapping to the pseudomolecules of Ae. tauschii and barley, SNPs 


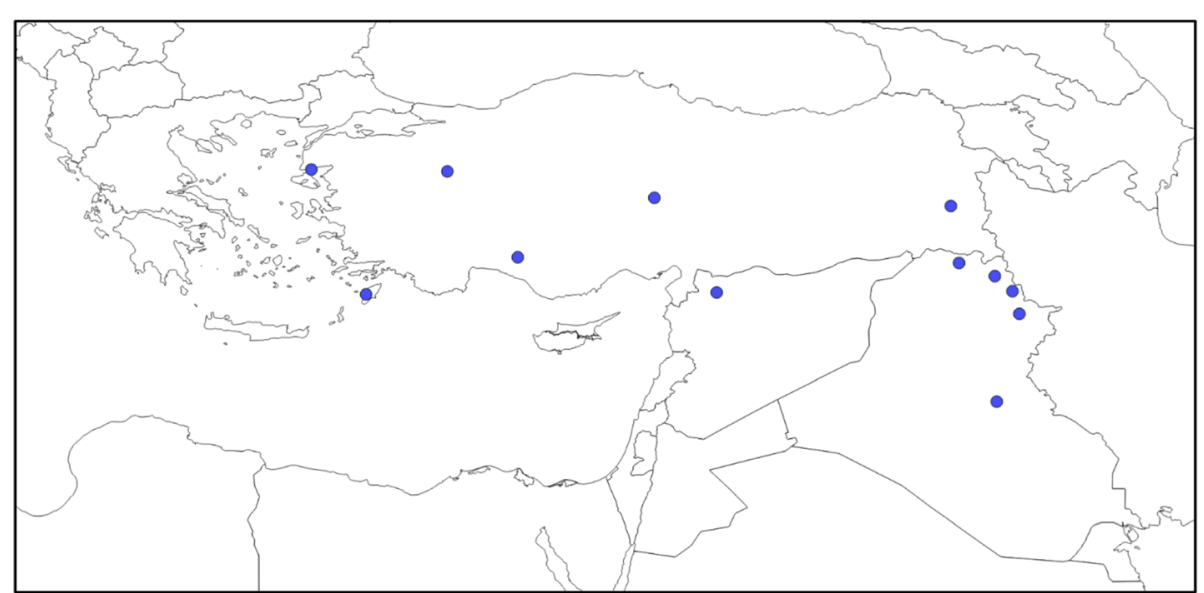

Fig. 1 The geographic distribution of the 12 tested accessions of Ae. umbellulata on the map of the northwestern part of the Middle East

and indels were anchored to the chromosomes. The distribution of SNPs and indels on barley and Ae. tauschii chromosomes were visualized using CIRCOS software [34] (Krzywinski et al. 2009).

\section{Development of markers and genotyping}

Indel markers were designed using indels longer than 3 bp that were anchored to the barley chromosomes. Primer sets were constructed with Primer3plus software [35]. To validate marker alleles, we genotyped $F_{1}$ hybrid from a cross between Ae. umbellulata accessions KU-4017 and KU-4043. Total DNA was extracted from leaves of $F_{1}$ plants and their parents. PCR was conducted using Quick Taq HD DyeMix (TOYOBO, Osaka, Japan). PCR products were resolved in $17 \%$ acrylamide gels, and

Table 1 List of Ae. umbellulata accessions used in this study

\begin{tabular}{|c|c|c|}
\hline $\begin{array}{l}\text { Accession } \\
\text { number }\end{array}$ & Origins & Locality \\
\hline$\overline{K U-4017}$ & Iraq & $\begin{array}{l}18.8 \mathrm{~km} \text { NNE from Sulaymaniyah to } \\
\text { Chuarta }\end{array}$ \\
\hline KU-4026 & Iraq & $25.9 \mathrm{~km}$ S from Kirkuk to Baghdad \\
\hline KU-4035 & Iraq & 5.5 km ENE from Koi Sanjak to Ranya \\
\hline KU-4043 & Iraq & SSW of Rowanduz \\
\hline KU-4052 & Iraq & $\begin{array}{l}4.4 \text { km NW from Amadiyah Mazorka } \\
\text { Gorge }\end{array}$ \\
\hline KU-4103 & Turkey & North of Van \\
\hline KU-5934 & Turkey & Suburbs of Kayseri \\
\hline KU-5954 & Turkey & Suburbs of Kutahia \\
\hline KU-8-7 & Turkey & Suburbs of Burdur (D) \\
\hline KU-12180 & Greece & $\begin{array}{l}5.1 \mathrm{~km} \text { W from Platania to Laerma } \\
\text { Rhodes }\end{array}$ \\
\hline KU-12198 & Greece & $\begin{array}{l}5.4 \mathrm{~km} \text { E from Mithymna to Madamados } \\
\text { Lesbos }\end{array}$ \\
\hline KU-8-5 & Syria & $\begin{array}{l}6 \mathrm{~km} \text { W of Qatana (Damascus - } \\
\text { Mt. Hermon) }\end{array}$ \\
\hline
\end{tabular}

the products were visualized under UV light after staining by ethidium bromide.

\section{Comparison of genetic diversity between Ae. umbellulata and Ae. tauschii}

The RNA-seq reads from the 10 Ae. tauschii accessions from the Transcriptome Shotgun Assembly division of DDBJ BioProject PRJDB4683 [16] were used for comparative analyses. We used the transcript sequences of Ae. tauschii KU-2075, which were constructed in our previous report [16], and Ae. umbellulata KU-4017 as reference transcripts. Quality control for the reads of $A e$. tauschii and T. urartu was performed using Trimmomatic version 0.33 [25] in the same way as for $A e$. umbellulata. The reads were aligned to the reference transcripts of Ae. tauschii KU-2075 and Ae. umbellulata KU-4017 using Bowtie 2 [28]. SNP calling was performed with SAMtools and Coval [29, 30] using the same criteria described above. SNPs that were assured of read depth $>10$ and no ambiguous nucleotides in any accessions were selected as high-confidence SNPs and used for analyzing intra- and interspecific variation. The number of segregating sites, Tajima's D statistic [36], and fixed nucleotide differences between species were estimated with DnaSP v5 software [37]. A neighbor-joining tree and a maximum likelihood tree were constructed based on the high-confidence SNPs. Bootstrap probability was calculated for 1000 replications.

\section{Estimation of orthologous transcripts of Ae. umbellulata and Ae. tauschii}

Orthologous pairs of the reference transcripts of $A e$. tauschii KU-2075 and Ae. umbellulata KU-4017 were estimated according to reciprocal best hits of BLAST analysis. A BLASTN search was performed using transcripts of Ae. tauschii KU-2075 as the queries against transcripts of Ae. umbellulata KU-4017, and vice versa. 
When the same best hit was detected and query coverage was over $80 \%$ in both BLAST analyses, the transcripts from Ae. umbellulata KU-4017 and Ae. tauschii KU-2075 were judged an orthologous pair.

\section{Gene expression analysis}

The mapped reads that were concordantly aligned to the reference transcripts were chosen from the alignment file with SAMtools [29]. Fragments per kilobase per million mapped reads (FPKM) values were calculated based on the concordantly mapped reads [38].

\section{Results}

\section{RNA sequencing of 12 Ae. umbellulata accessions}

To evaluate genetic diversity based on a large number of DNA polymorphisms in the U-genome species Ae. umbellulata, RNA-seq was performed on the 12 representative accessions, generating 3.5-6.1 million paired-end reads per one accession (Table 2). These reads were analyzed according to the workflow shown in Additional file 1: Figure S1. After filtering out reads with low quality, 2.2-3.9 million paired-end reads (56.2$74.1 \%)$ were obtained. Due to the absence of a reference genome for Ae. umbellulata, transcript sequences for each of the 12 accessions were constructed by de novo assembly of the filtered reads. For each accession, 20,996 to 59,253 transcripts with N50 values of 899 to 1365 bp were deduced. One isoform was chosen as a unigene if a transcript had multiple isoforms. Finally, 12 sets of unigenes composed of 20,675 to 55,831 representative isoforms were obtained (Table 2) and used as reference transcript datasets for pairwise alignments between the accessions.

\section{Genome-wide identification of SNPs and indels in Ae. umbellulata}

To detect SNPs and indels among the accessions, the filtered reads of each accession were aligned to the reference transcripts of all other accessions, and SNPs and indels were called according to the thresholds with read depth $>10$. SNPs and indels identified from comparisons of the same accessions were regarded as artifacts. After filtering to remove these putative artifacts, 2925-44,751 SNPs and 77-1389 indels were obtained among the accessions (Table 3). The maximum numbers of SNPs and indels were obtained between KU-4035 and KU-12180 (44,751 SNPs and 1389 indels), with the minimum between KU-4017 and KU-4026 (2925 SNPs and 77 indels).

Table 2 Summary of RNA sequencing for 12 accessions of Ae. umbellulata

\begin{tabular}{|c|c|c|c|c|c|c|}
\hline Accession & $\begin{array}{l}\text { Read } \\
\text { pairs }\end{array}$ & $\begin{array}{l}\text { Filtered } \\
\text { read pairs }\left(\%{ }^{\mathrm{a}}\right)\end{array}$ & (unigenes) & $\begin{array}{l}\mathrm{N} 50 \\
\text { (bp) }\end{array}$ & $\begin{array}{l}\text { Median contig } \\
\text { length } \\
\text { (bp) }\end{array}$ & $\begin{array}{l}\text { Total assembled } \\
\text { bases } \\
\text { (Mbp) }\end{array}$ \\
\hline KU-4017 & $3,738,403$ & $2,515,683(67.29 \%)$ & $\begin{array}{l}39,359 \\
(37640)\end{array}$ & 1238 & 658 & 36 \\
\hline KU-4026 & $4,935,634$ & $3,429,252(69.48 \%)$ & $\begin{array}{l}20,996 \\
(20675)\end{array}$ & 900 & 578.5 & 15.6 \\
\hline KU-4035 & $6,077,268$ & $3,890,911(64.02 \%)$ & $\begin{array}{l}57,029 \\
(52216)\end{array}$ & 1350 & 654 & 52.9 \\
\hline KU-4043 & $4,090,151$ & 2,392,708 (58.50\%) & $\begin{array}{l}50,985 \\
(48590)\end{array}$ & 1095 & 518 & 38.9 \\
\hline KU-4052 & $3,829,992$ & $2,152,503$ (56.20\%) & $\begin{array}{l}47,320 \\
(44869)\end{array}$ & 1179 & 577 & 39.4 \\
\hline KU-4103 & $3,875,477$ & $2,506,545(64.68 \%)$ & $\begin{array}{l}31,418 \\
(30873)\end{array}$ & 899 & 507 & 22.1 \\
\hline KU-5934 & $5,114,283$ & $3,507,234(68.58 \%)$ & $\begin{array}{l}57,466 \\
(52751)\end{array}$ & 1332 & 630 & 52.7 \\
\hline KU-5954 & $3,686,807$ & $2,694,916(73.10 \%)$ & $\begin{array}{l}45,164 \\
(41780)\end{array}$ & 1365 & 699 & 44.1 \\
\hline KU-12180 & $3,455,952$ & $2,560,779(74.10 \%)$ & $\begin{array}{l}46,323 \\
(44000)\end{array}$ & 1229 & 631 & 41.2 \\
\hline KU-12198 & $3,623,492$ & $2,666,981(73.60 \%)$ & $\begin{array}{l}50,069 \\
(46178)\end{array}$ & 1359 & 655 & 47,7 \\
\hline KU-8-5 & $3,669,766$ & $2,699,452(73.56 \%)$ & $\begin{array}{l}52,648 \\
(48981)\end{array}$ & 1259 & 654 & 48.2 \\
\hline KU-8-7 & $3,798,153$ & $2,524,378(66.46 \%)$ & $\begin{array}{l}59,253 \\
(55831)\end{array}$ & 1160 & 524 & 46.9 \\
\hline
\end{tabular}


$\stackrel{\Upsilon}{z}$

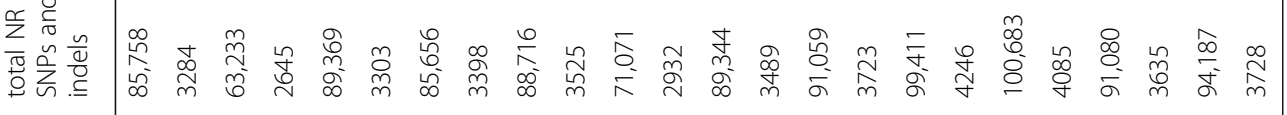

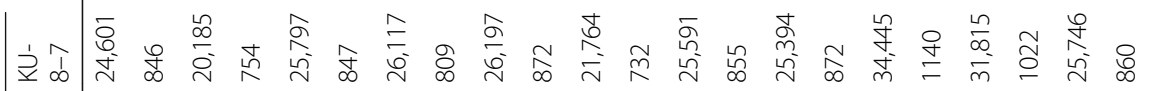

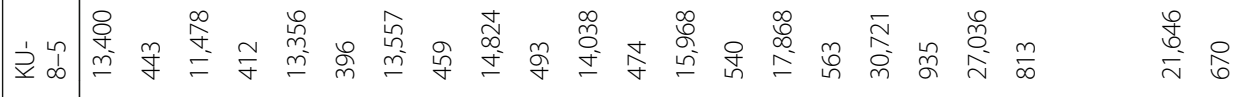

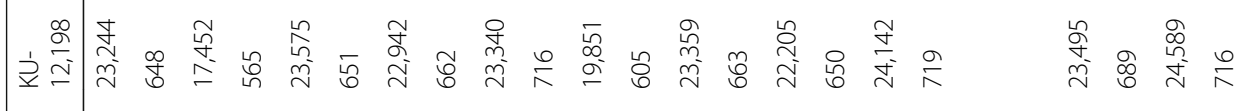

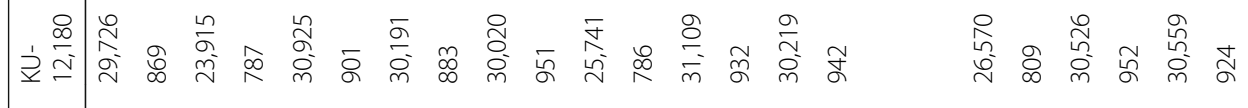

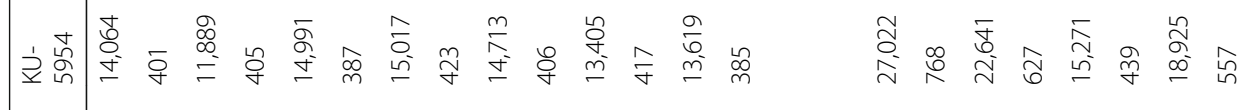

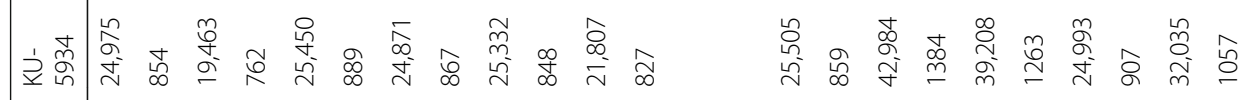

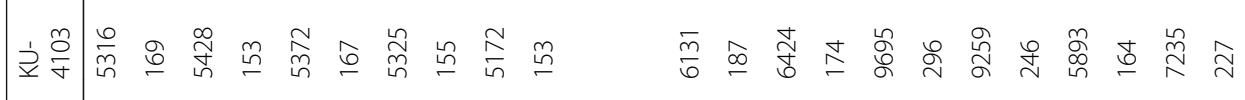

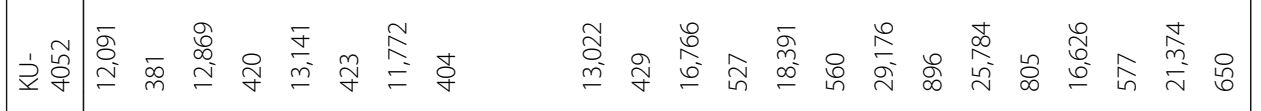

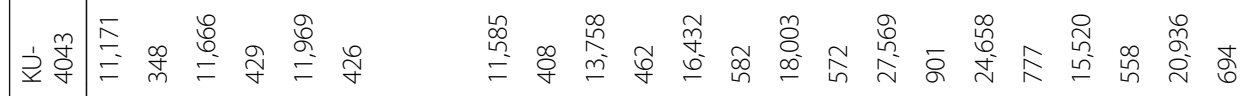

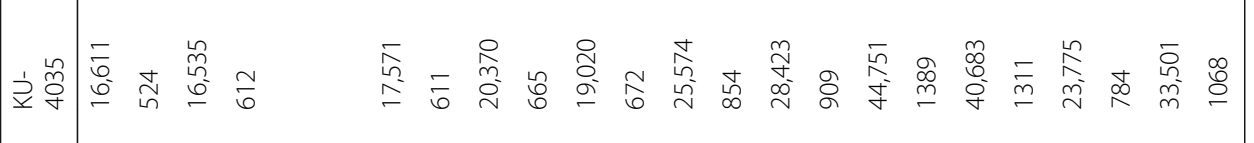

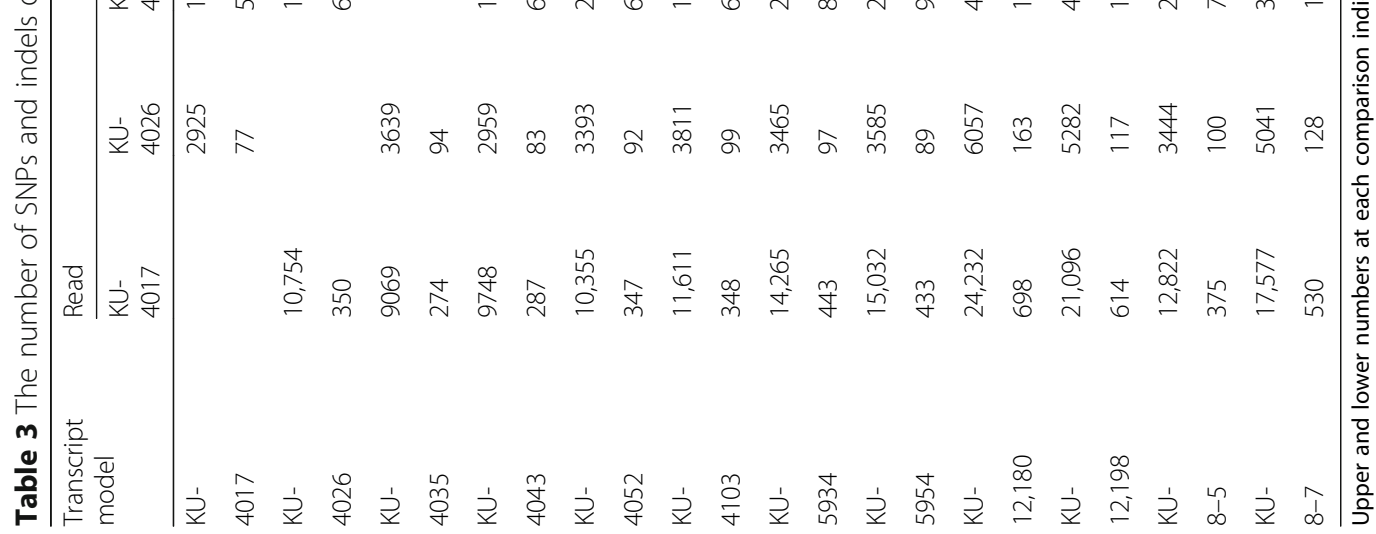


For efficient use of the identified SNPs and indels as genetic markers, their chromosomal locations must be known. Here, we used the Ae. tauschii and barley pseudomolecules as virtual chromosomes of Ae. umbellulata, and mapped the unigene sequences of the Ae. umbellulata reference transcript datasets to the Ae. tauschii and barley chromosomes. In the reference transcripts, $75.87-85.35 \%$ of the unigenes were mapped to $A e$. tauschii and 52.08-67.69\% to barley chromosomes (Additional file 1: Table S1). Based on the positional information of the mapped unigenes, SNPs and indels were anchored to the chromosomes of both species. In any pairwise comparison between Ae. umbellulata accessions, $81.83-89.50 \%$ of SNPs and $75.28-89.26 \%$ of indels were anchored to Ae. tauschii chromosomes, while $63.17-75.16 \%$ of SNPs and $59.04-77.78 \%$ of indels were anchored to barley chromosomes (Additional file 1: Tables S2, S3). The distribution of SNPs over each chromosome of Ae. tauschii and barley was visualized with CIRCOS [34] for the Ae. umbellulata accession pairs with the maximum or minimum number of SNPs. The SNPs covered all chromosomes (Fig. 2).

Non-redundant SNPs and indels were estimated for each of the 12 sets of reference transcripts. A total of 63,233-100,683 non-redundant SNPs and 2645-4246 non-redundant indels were detected in the tested $A e$. umbellulata accessions (Table 3). On average, 73,075 non-redundant SNPs (85.07\%) were anchored to $A e$. tauschii chromosomes, and 58,247 (70.40\%) non-redundant SNPs to barley chromosomes (Additional file 1: Tables S4, S5). The smallest number of anchored non-redundant SNPs was observed on chromosomes 4D in Ae. tauschii and 4H in barley (Fig. 3). Each chromosome of Ae. tauschii and barley had an average of 10,439 and 8321 non-redundant SNPs, respectively. The anchored non-redundant SNPs were distributed over all seven chromosomes of Ae. tauschii and barley (Fig. 2).

We estimated the percentages of non-redundant SNPs anchored to the Ae. tauschii chromosomes overlapped those on barley chromosomes (Fig. 4). Venn diagrams showed that $69.18 \%$ of non-redundant SNPs were anchored to both Ae. tauschii and barley chromosomes. The percentage of non-redundant SNPs uniquely anchoring to Ae. tauschii chromosomes was $24.96 \%$. Only $5.86 \%$ of non-redundant SNPs were uniquely anchored to barley chromosomes. After integration of these anchored non-redundant SNPs, 77,625 non-redundant SNPs were placed on the chromosomes.

\section{Application of indel markers to confirmation of $F_{1}$ formation}

To confirm usefulness of the identified polymorphisms as genetic markers, primer sets for 27 indels were designed. The indel markers were applied to genotype $F_{1}$ hybrid from a cross between two Ae. umbellulata accessions, KU-4017 and KU-4043; nine markers enabled detection of the genetic differences between the accessions and confirmed their $F_{1}$ formation (Additional file 1: Figure S2). The difference in amplicon size between the parents was observed in the five markers. Presence/absence of amplicons between the parents was detected in the two markers. In the other two markers, the parents were distinguished by an extra band.

\section{Comparison of genetic diversity in Ae. umbellulata and Ae. tauschii}

Ae. tauschii is widely distributed over central Eurasia and has three divergent lineages, TauL1, TauL2 and TauL3 [39]. On the other hand, the habitat of Ae. umbellulata is limited to West Asia. To examine how differences in geographic distribution and evolutionary history of these species affected the extent of DNA polymorphisms and the distribution of allele frequency, genetic diversity in Ae. umbellulata and Ae. tauschii was evaluated with SNPs deduced using the same RNA-seq platform. To compare intraspecific diversity of the two Aegilops species, reads from RNA-seq of the 12 Ae. umbellulata accessions, the 10 Ae. tauschii accessions [16] and T. urartu KU-199-5 were aligned to the reference transcripts of Ae. umbellulata KU-4017. T. urartu KU-199-5 was used as an outgroup species. To elucidate the phylogenetic relationship of Ae. umbellulata and Ae. tauschii accessions, maximum likelihood and neighbor-joining trees were constructed based on the high-confidence SNPs (Fig. 5; Additional file 1: Figure S3a). The three species were clearly separated, with the Aegilops species more closely related than $T$. urartu, with fixed nucleotide differences between Ae. umbellulata and Ae. tauschii smaller than those between Ae. tauschii and T. urartu or between Ae. umbellulata and T. urartu (Additional file 1: Table S6). The external branches of Ae. umbellulata were longer than those of Ae. tauschii. Ae. umbellulata KU-12180 was isolated from the other accessions, supporting observations from the phylogenetic trees constructed based on nucleotide polymorphisms in a small number of genes [3]. However, the clear divergent lineages observed in Ae. tauschii were not found in the Ae. umbellulata accessions (Fig. 5). When the reference transcripts of Ae. tauschii KU-2075 was used for the alignments and SNP calling, similar results were obtained (Additional file 1: Table S6, Figures S3b, S4).

The number of segregating sites in Ae. umbellulata was larger than in Ae. tauschii (Table 4), indicating that Ae. umbellulata has relatively high genetic diversity. To test how differences in habitat and evolutionary history between Ae. umbellulata and Ae. tauschii affected allele frequency distribution in these two species, the derived allele frequency distribution for each species was estimated using T. urartu as an outgroup species (Fig. 6). At 

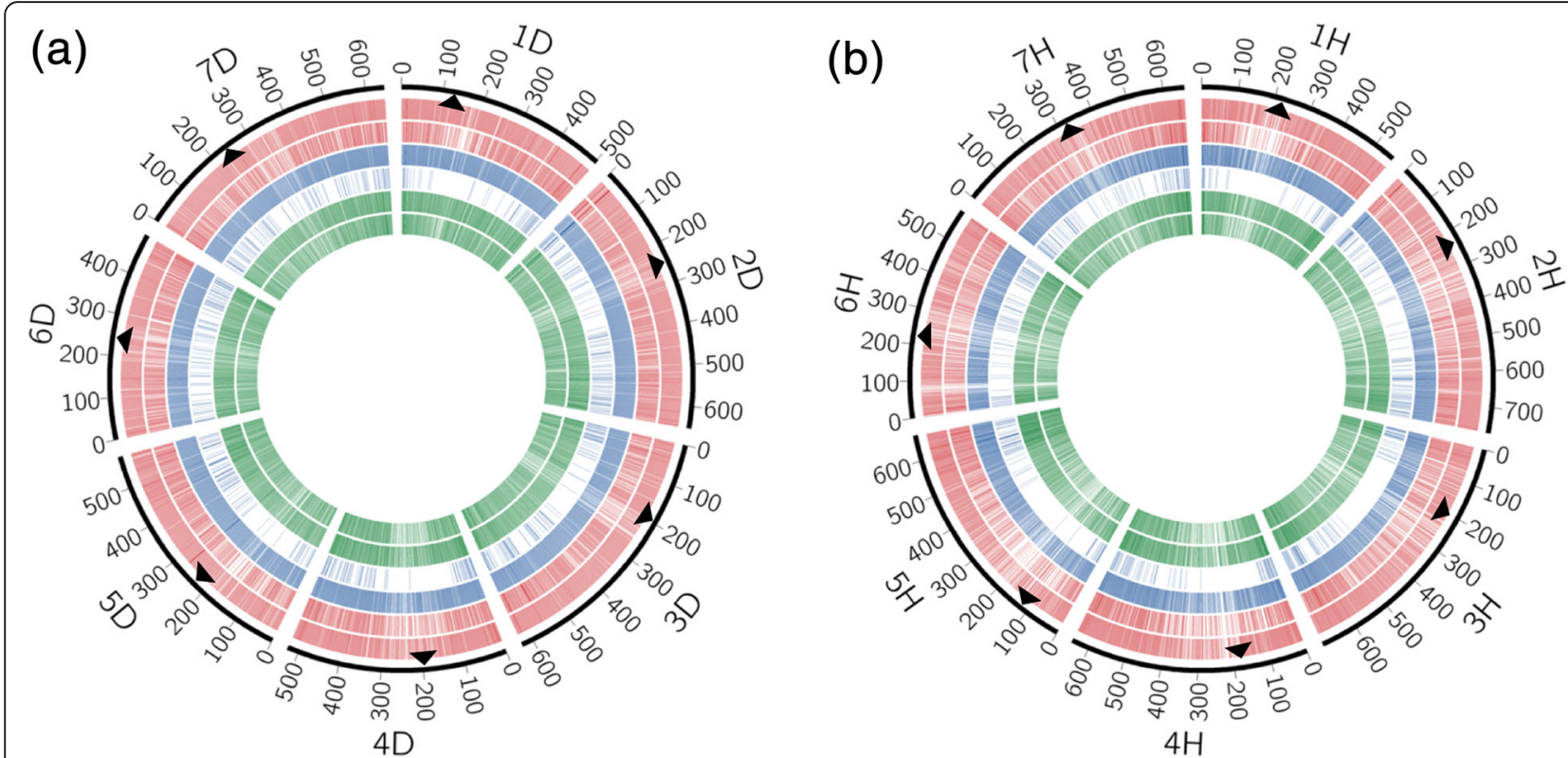

Fig. 2 Distribution of transcripts and SNPs detected in pairwise comparisons of Ae. umbellulata accessions on the physical map of Ae. tauschii (a) and barley (b); scale in Mb. The three circles of the same color show the number of transcripts and SNPs from outer to inner circles. The red circles indicate the richest SNP pairs (KU-4035 read-mapped to KU-12180 transcript). The blue circles indicate the least rich pairs (KU-4026 readmapped to KU-4017 transcript) in each combination of lineages. The green circles indicate the non-redundant SNPs mapped against KU-4043 transcripts. Arrowheads represent centromeric positions of each chromosome

a polymorphic site, a nucleotide that is inconsistent with that of outgroup species is defined as a derived allele, because this allele is considered to be newly generated by a mutation in population of the tested species [40]. The derived allele frequency distributions of the two species showed distinct patterns. Alleles with intermediate frequency were predominantly detected in $A e$. tauschii, while alleles with rarer frequency were more common in Ae. umbellulata. As expected from the difference in the allele frequency distributions, Tajima's D statistic [36] for Ae. tauschii and Ae. umbellulata respectively gave positive and negative values (Table 4).

Nucleotide diversity $(\theta)$ [41] in Ae. umbellulata and Ae. tauschii was estimated for each transcript. The $\theta$ value for each transcript of Ae. umbellulata was weakly correlated with that of Ae. tauschii (Figs. 7a, b: Kendall's rank correlation $\tau=-0.026$ and 0.043 ). To avoid the possibility of bias due to differences in the accuracy and efficiency of short read alignments between intra- and interspecies, we compared $\theta$ for the 6062 orthologous

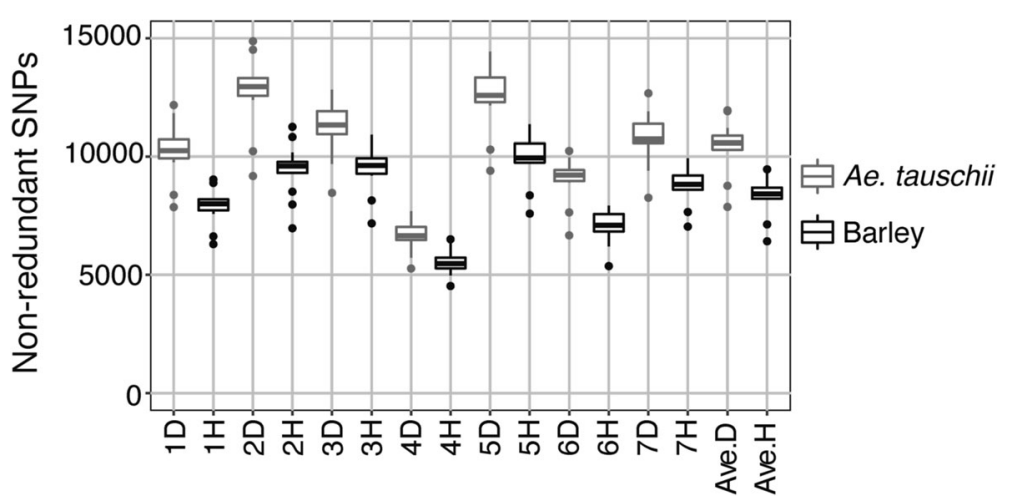

Fig. 3 Boxplots of non-redundant SNPs anchored to chromosomes of Ae. tauschii (gray) and barley (black). The number of non-redundant SNPs on the chromosomes for each reference transcript dataset is plotted. Ave.D and Ave.H respectively indicate average number of non-redundant SNPS per chromosome when genomes of Ae. tauschii and barley were used 


\section{Non-redundant SNPs}

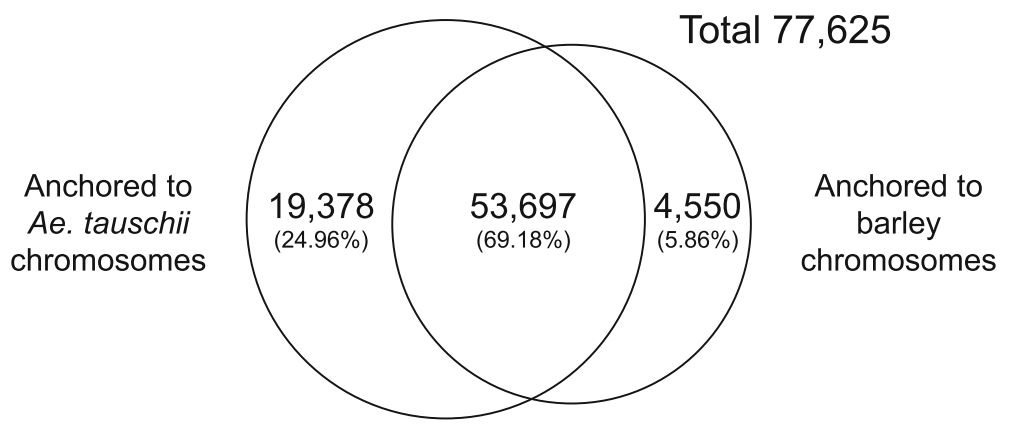

Fig. 4 Venn diagrams of non-redundant SNPs anchored to the Ae. tauschii and barley chromosomes. The numbers indicate mean values of nonredundant SNPs and indels derived from each of the reference transcript datasets

pairs between Ae. umbellulata and Ae. tauschii. These pairs were retrieved by reciprocal best hits of BLAST analysis between the reference transcript datasets of $A e$. umbellulata KU-4017 and Ae. tauschii KU-2075. This approach enables evaluation of genetic diversity using only the $\theta$ value based on SNPs derived from the intraspecies alignments of reads. Although gene expression of the orthologous pairs showed a relatively strong correlation (Fig. 7c: $\tau=0.577$ ), the values of $\theta$ between the pairs designated a weak correlation (Fig. $7 \mathrm{~d}$ : $\mathrm{\tau}=$ 0.049). Taken together, the reproducible observations from different approaches underpin the distinct extent

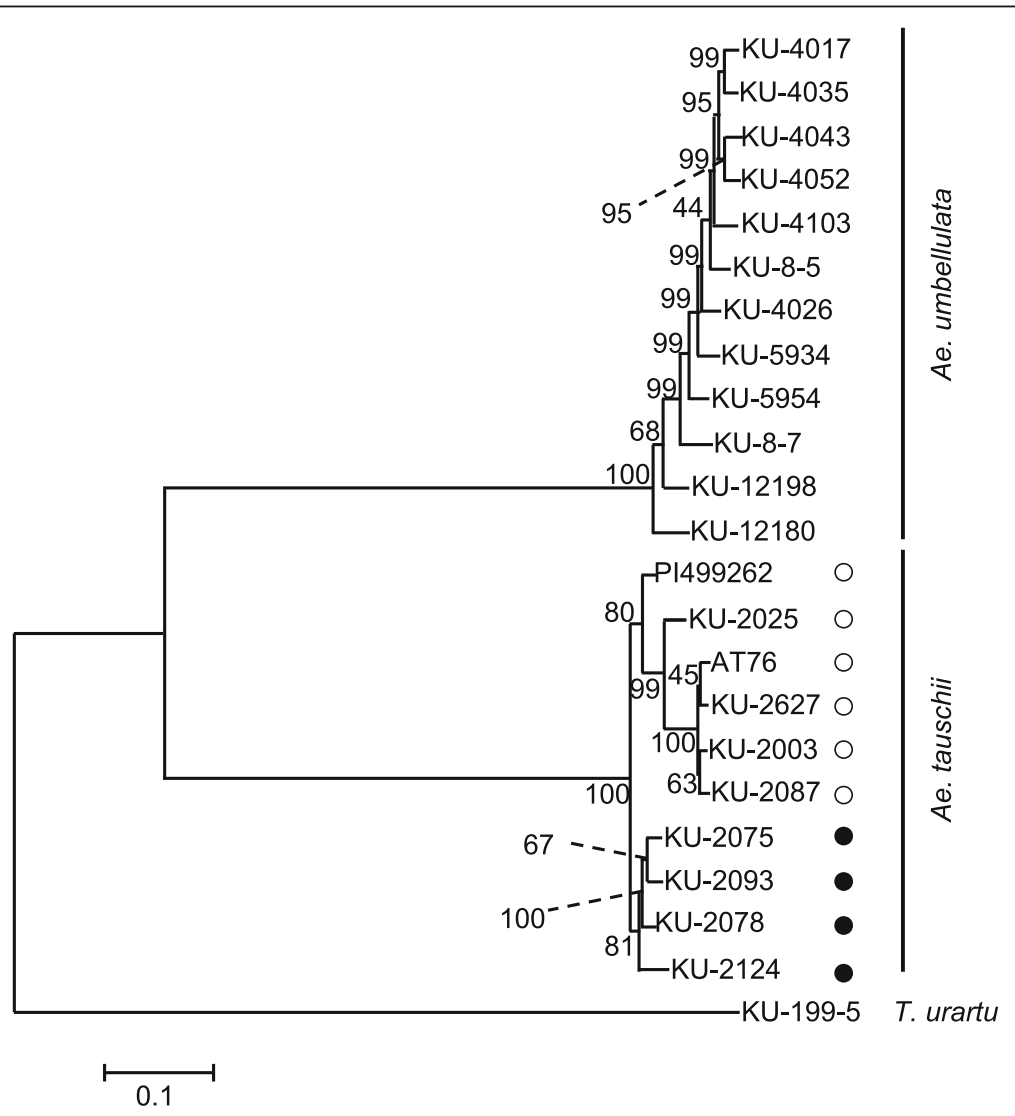

Fig. 5 Phylogenetic relationship between the 12 Ae. umbellulata accessions, 10 Ae. tauschii accessions and one T. urartu accession based on SNPS estimated using the Ae. umbellulata KU-4017 reference transcript dataset. The tree was constructed by the maximum-likelihood method. The bootstrap values (1000 replicates) are shown on each branch. White and black circles of Ae. tauschii respectively correspond to the divergent lineages TauL1 and TauL2 [39]. T. urartu was used as the outgroup species 
Table 4 Summary of nucleotide polymorphisms in Ae. umbellulata and Ae. tauschii

\begin{tabular}{|c|c|c|c|c|}
\hline \multirow{2}{*}{$\begin{array}{l}\text { Reference } \\
\text { SNPS }\end{array}$} & \multicolumn{2}{|c|}{ Ae. umbellulata KU-4017 } & \multicolumn{2}{|c|}{ Ae. tauschii KU-2075 } \\
\hline & Ae. umbellulata & Ae. tauschii & Ae. umbellulata & Ae. tauschi \\
\hline \# of accession & 12 & 10 & 12 & 10 \\
\hline \# of site & 31,677 & 31,677 & 29,702 & 29,702 \\
\hline \# of segregating site & 4751 & 4136 & 4301 & 3832 \\
\hline singleton & 1992 & 1103 & 1759 & 1019 \\
\hline non-singleton & 2759 & 3033 & 2542 & 2813 \\
\hline Tajima's D & -0.24 NS & $0.86 \mathrm{NS}$ & $-0.22 \mathrm{NS}$ & 0.89 NS \\
\hline
\end{tabular}

of nucleotide polymorphisms between Ae umbellulata and Ae. tauschii at the gene level.

\section{Discussion}

RNA-seq is a powerful approach to identify novel genetic markers for Triticeae species

To identify genome wide polymorphisms (SNPs and indels) and to develop novel genetic markers, we conducted 300-bp paired-end RNA sequencing of leaf tissues from 12 representative Ae. umbellulata accessions using the Illumina MiSeq platform. By using Ae. tauschii and barley pseudomolecules as the virtual chromosomes of Ae. umbellulata due to the conserved synteny between Triticeae species [23, 24], an average of 73,075 and 58,247 non-redundant SNPs in Ae umbellulata were successfully anchored to the chromosomes of Ae. tauschii and barley, respectively (Fig. 3; Additional file 1: Tables S4, S5). The application of reference-quality genome sequences of Ae. tauschii [21] dramatically improved the number of SNPs anchored to the chromosomes compared with a previous study [16], in which SNPs in Ae. tauschii were linked to the chromosomes by combining the draft genome sequences of Ae. tauschii [42] with its genetic linkage map [43]. Even when SNPs in Ae. tauschii were mapped to Ae. tauschii chromosomes, the number of anchored SNPs was slightly smaller than when the SNPs were mapped to the chromosomes of barley with reference-quality genome sequences [16]. The elaboration of SNP anchoring enabled capturing an average of 10,439 non-redundant SNPs per chromosome (Fig. 3; Additional file 1: Table S5), which were well distributed over each chromosome (Fig. 2). Since polymorphisms derived RNA-seq data were composed of only SNPs and indels in exons and untranslated regions of the expressed genes, the RNA-seq-based approach avoided the repetitiveness of intergenic regions and much of the genome complexity, resulting in identification of a large number of SNPs anchored to the virtual chromosomes. Recently, a high-density consensus linkage map including 3009 SNP markers derived from genotyping-by-sequencing was constructed in two biparental populations from four accessions of Ae. umbellulata [9].
The RNA-seq approach fills the gaps left by other genotyping methods such as genotyping-by-sequencing when developing genetic markers for Triticeae species without a genome sequence, such as Ae. umbellulata.

In our RNA-seq-based approach, the identified SNPs and indels were arranged on the Ae. umbellulata chromosomes in an order reflecting the conserved synteny with Ae. tauschii and barley (Fig. 2). When a genetic map is constructed using these anchored SNPs and indels, changes in the marker order should be considered carefully due to the existence of chromosomal rearrangements in Ae. umbellulata. Structural rearrangements have been observed for Ae. umbellulata chromosomes when the order of genetic markers was compared among Ae. umbellulata, Ae. tauschii and common wheat [9, 44-46]. For example, chromosome $4 \mathrm{U}$ has segmental homoeology to the group 6 chromosomes of common wheat [46]. Similarly, partial segments of chromosome $6 \mathrm{U}$ have homoeology to hexaploid wheat group 4 and 5 chromosomes [9]. These observations support the occurrence of structural rearrangements such as translocation in Ae. umbellulata.

The power of indel detection with RNA-seq is not as high as that of SNPs, because indels in exons often have functionally deleterious effects on proteins and are purged from the genome by purifying selection. Notwithstanding this disadvantage, RNA-seq still provides useful indel markers for genetic mapping [47]. The indel markers were effective for validating detection of $F_{1}$ alleles between Ae. umbellulata KU-4017 and KU-4043 (Additional file 1: Figure S2). These markers would allow rough map construction.

\section{Contrasting patterns of nucleotide diversity between $A e$. umbellulata and Ae. tauschii}

Differences in the habitats, morphology, population structure and phenological traits between Ae. tauschii and Ae. umbellulata may result in differences in the pressures of natural selection and the effect of genetic drift on genes, shaping the extent of DNA polymorphisms and allele frequency distribution between the species. In spite of the limited habitats of Ae. 

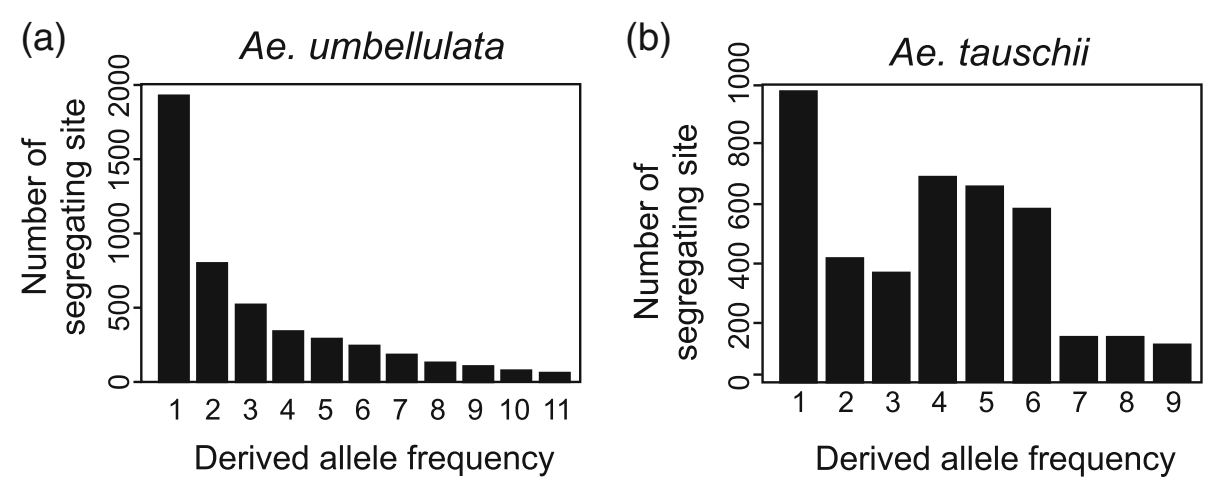

Fig. 6 Derived allele frequency distribution in Ae. umbellulata $(n=12)(\mathbf{a})$ and Ae. tauschii $(n=10)(\mathbf{b})$, respectively. Ae. umbellulata KU-4017 transcripts were used as the reference. Derived alleles were estimated using the outgroup species $T$. urartu

umbellulata, the present study showed that Ae. umbellulata has higher genetic diversity than the more widely distributed species Ae. tauschii (Fig. 5; Table 4). This observation is consistent with a previous report [48], in which intra- and interspecific genetic variation in seven diploid Aegilops species was evaluated using amplified fragment length polymorphisms, also concluding that genetic diversity in Ae. umbellulata is higher than in Ae. tauschii. Our comparative analyses showed no clear lineage differentiation in Ae. umbellulata (Fig. 5; Additional file 1:
Figures S3, S4) and the prevalence of alleles with rarer frequencies (Fig. 6; Additional file 1: Figure S5), implying that the alleles with rarer frequencies are the main source of the genetic diversity observed in Ae. umbellulata.

The longer external branches of the phylogenetic tree in Ae. umbellulata suggest higher genetic differentiation of each Ae. umbellulata accession than Ae. tauschii (Fig. 5; Additional file 1: Figures S3, S4). Generally self-pollination inhibits gene flow via pollen, increasing genetic differentiation among local populations [49]. Since Ae. umbellulata

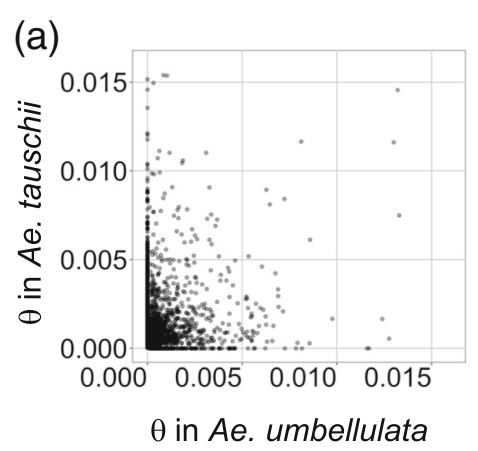

(c)

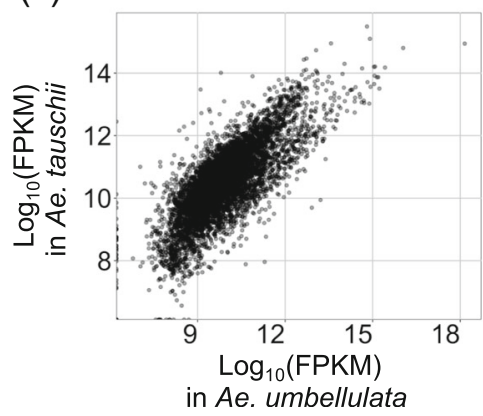

(b)

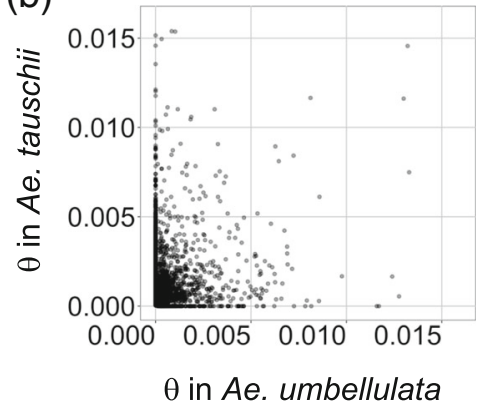

(d)

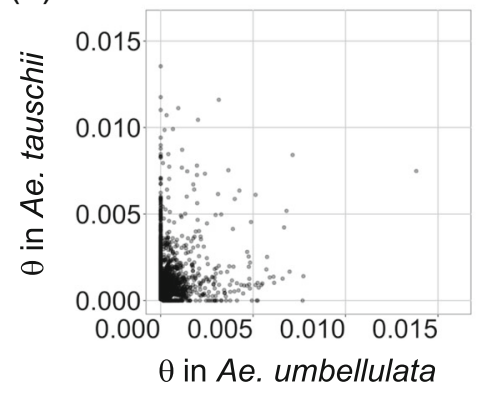

Fig. 7 Scatter plot of nucleotide diversity $(\theta)$ in Ae. umbellulata $(n=12)$ and Ae. tauschii $(n=10)$ when 7666 transcripts derived from Ae. umbellulata KU-4017 were used as the references (a) and when 6622 transcripts derived from Ae. tauschii KU-2075 were used as the references (b). For both sets of transcripts, at least one of the species had polymorphisms. Scatter plot of gene expression (FPKM) in the 6062 orthologous pairs of Ae. umbellulata KU-4017 and Ae. tauschii KU-2075 retrieved by reciprocal best hits of BLAST analyses (c). Scatter plot of $\theta$ in the 3354 orthologous pairs of Ae. umbellulata KU-4017 and Ae. tauschii KU-2075 that had polymorphisms in one of the species (d). Kendall's rank correlation $(\tau)$ for $(\mathbf{a}),(\mathbf{b}),(\mathbf{c})$, and $(\mathbf{d})$ was $-0.026(p=0.0033), 0.043(p=5.3 e-6), 0.577(p<2.2 \mathrm{e}-16)$ and $0.049(p=0.0002)$, respectively 
is a self-fertilizing plant, this general view could be applicable to the observed genetic differentiation between the accessions of Ae. umbellulata. Considering Ae. tauschii is also a self-fertilizing species, another factor may contribute to shaping the distinct patterns of nucleotide polymorphism in these two species. If the time of expansion and colonization into the modern habitats differed between species, neutral mutations are expected to have accumulated more within a local population of the species with the earlier expansion and colonization, generating genetic differentiation between local populations under the limited gene flow. If this hypothesis is accepted, the time of expansion and colonization into the modern habitat of Ae. umbellulata is presumed to be older than that of Ae. tauschii. These different evolutionary scenarios and habitats of Ae. tauschii and Ae. umbellulata are likely to have shaped distinct genetic diversity for each gene from their common ancestor. The scatter plots of nucleotide diversity in the transcripts of Ae. umbellulata and Ae. tauschii show weaker correlations between the orthologous pairs (Fig. 7), suggesting that genes of Ae. umbellulata were subjected to natural selection pressure and effects of genetic drift that were distinct from those of $A e$. tauschii. Future larger-scale population genomic analyses in both species will disclose population dynamics with higher resolution and more powerfully detect footprints of natural selection in each gene.

\section{Conclusion}

The RNA-seq-based approach is efficient for development of a large number of molecular markers and for conducting population genetic analyses for a large number of genes in wheat wild relatives such as Ae. umbellulata lacking genomic information. In addition, Ae. umbellulata, harboring relatively high genetic diversity, has considerable potential as a genetic resource for breeding of common wheat.

\section{Additional file}

Additional file 1 : Table S1. Summary of the number of unigenes anchored to barley and Ae. tauschii genome. Table S2. The number of SNPs and indels anchored to the chromosomes of Ae. tauschii out of the SNPS and indels detected in each transcript-read pairing of $12 \mathrm{Ae}$. umbellulata accessions. Table S3. The number of SNPs and indels anchored to the barley chromosomes out of the SNPS and indels detected in each transcript-read pairing of 12 Ae. umbellulata accessions. Table S4. The number of non-redundant SNPs anchored to each Ae. tauschii chromosome. Table S5. The number of non-redundant SNPS anchored to each barley chromosome. Table S6. Summary of nucleotide polymorphism and divergence in Ae. umbellulata, Ae. tauschii and T. urartu. Figure S1. The workflow of RNA-seq analysis. Figure S2. Images of polyacrylamide gel electrophoresis for indel markers. Figure S3. Phylogenetic relationship between $12 \mathrm{Ae}$. umbellulata accessions, 10 Ae. tauschii accessions and one T. urartu accession based on SNPs that was estimated by using the Ae. umbellulata KU-4017 reference transcript dataset (a) and the Ae. tauschii KU-2075 reference transcript dataset (b). These trees were constructed by Neighbor-Joining method. Figure S4. Phylogenetic relationship between the $12 \mathrm{Ae}$. umbellulata accessions, $10 \mathrm{Ae}$. tauschii accessions and one $T$. urartu accession based on SNPs estimated using the Ae. tauschii KU-2075 reference transcript dataset. The tree was constructed by the maximum-likelihood method. Figure S5. Derived allele frequency distribution in Ae. umbellulata $(n=12)$ (a) and Ae. tauschii $(n=10)(b)$, respectively. Ae. tauschii KU-2075 transcripts were used as the reference. Derived alleles were estimated using the outgroup species T. urartu. (PDF $824 \mathrm{~kb}$ )

\section{Abbreviations}

FPKM: Fragments per kilobase per million mapped reads; indels: Insertions and deletions; RNA-seq: RNA-sequencing; SNPs: Single nucleotide polymorphisms

\section{Acknowledgments}

The Ae. umbellulata seeds used in this study were supplied by the National BioResource Project-Wheat, Japan (www.nbrp.jp). Computations for RNA sequence assembly and alignments of reads were performed on the NIG supercomputer at the ROIS National Institute of Genetics, Japan.

\section{Funding}

This work was supported by Grant-in-Aid for Scientific Research on Innovative Areas No. 17H05842, by Grant-in-Aid for Scientific Research (B) No. $16 \mathrm{H} 04862$ to ST from the Ministry of Education, Culture, Sports, Science and Technology (MEXT) of Japan, and by MEXT as part of a Joint Research Program implemented at the Institute of Plant Science and Resources, Okayama University, Japan. KY was supported by JST, PRESTO (No. JPMJPR15QB).

\section{Availability of data and materials}

The datasets generated during and/or analyzed during the current study are available from the corresponding author on reasonable request.

\section{Authors' contributions}

KY, KS and ST designed the whole project. MO, KY, KS and ST wrote the manuscript. MO, AM, and YM performed experiments. MO, RN, and $K Y$ conducted RNA-sequencing analyses. All authors read and approved the final manuscript.

\section{Ethics approval and consent to participate}

Not applicable

\section{Consent for publication}

Not applicable

\section{Competing interests}

The authors declare that they have no conflicts of interest.

\section{Publisher's Note}

Springer Nature remains neutral with regard to jurisdictional claims in published maps and institutional affiliations.

\section{Author details}

'Graduate School of Agricultural Science, Kobe University, Rokkodai 1-1, Nada-ku, Kobe 657-8501, Japan. ${ }^{2}$ Institute of Plant Science and Resources, Okayama University, Kurashiki, Japan.

Received: 29 January 2018 Accepted: 25 October 2018

Published online: 08 November 2018

\section{References}

1. Lilienfeld FA. H. Kihara: genome-analysis in Triticum and Aegilops. X. Concluding review. Cytologia. 1951;16:101-23.

2. Wang GZ, Miyashita NT, Tsunewaki K. Plasmon analyses of Triticum (wheat) and Aegilops: PCR-single strand conformational polymorphism (PCR-SSCP) analyses of organellar DNAs. Proc Natl Acad Sci U S A. 1997;94:14570-7.

3. Okada M, Yoshida K, Takumi S. Hybrid incompatibilities in interspecific crosses between tetraploid wheat and its wild diploid relative Aegilops umbellulata. Plant Mol Biol. 2017;95:625-45. 
4. Kimber $\mathrm{G}$. The addition of the chromosomes of Aegilops umbellulata to Triticum aestivum (var. Chinese spring). Genet Res. 1967;9:111-4.

5. Law CN, Payne PI. Genetical aspects of breeding for improved grain protein content and type in wheat. J Cereal Sci. 1983;1:79-93.

6. Chhuneja P, Kaur S, Goel RK, Aghaee-Sarbaezeh M, Parashar M, Dhaliwal HS. Transfer of leaf rust and stripe rust resistance from Aegilops umbellulata Zhuk. To bread wheat (Triticum aestivum L.). Genet Resour Crop Evol. 2008; 55:849-59.

7. Edae EA, Olivera PD, Jin Y, Poland JA, Rouse MN. Genotype-by-sequencing facilitates genetic mapping of a stem rust resistance locus in Aegilops umbellulata, a wild relative of cultivated wheat. BMC Genomics. 2016;17: 1039.

8. Bansal M, Kaur S, Dhaliwal HS, Bains NS, Bariana HS, Chhuneja P, Bansal UK. Mapping of Aegilops umbellulata-derived leaf rust and stripe rust resistance loci in wheat. Plant Pathol. 2017:66:38-44.

9. Edae EA, Olivera PD, Jin Y, Rouse MN. Genotyping-by-sequencing facilitates a high-density consensus linkage map for Aegilops umbellulata, a wild relative of cultivated wheat. G3. 2017;7:1551-61.

10. Wang J, Wang C, Zhen S, Li X, Yan Y. Low-molecular-weight glutenin subunits from the $1 \mathrm{U}$ genome of Aegilops umbellulata confer superior dough rheological properties and improve bread making quality of bread wheat. J Sci Food Agric. 2017. https://doi.org/10.1002/jsfa.8700.

11. Schachermayr G, Siedler H, Gale MD, Winzeler H, Winzeler M, Keller B. Identification and localization of molecular markers linked to the Lr9 leaf rust resistance gene of wheat. Theor Appl Genet. 1994;88:110-5.

12. Brown JWS, Kemble RJ, Law CN, Flavell RB. Control of endosperm proteins in Triticum aestivum (ver. Chinise spring) and Aegilops umbellulata by homeologous group 1 chromosomse. Genetics. 1979;93:189-200.

13. Fox SE, Geniza M, Hanumappa M, Naithani S, Sullivan C, Preece J, Tiwari VK, Elser J, Leonard JM, Sage A, Gresham C, Kerhornou A, Bolser D, McCarthy F, Kersey P, Lazo GR, Jaiswal P. De novo transcriptome assembly and analyses of gene expression during photomorphogenesis in diploid wheat Triticum monococcum. PLoS One. 2014;9:e96855.

14. lehisa JCM, Shimizu A, Sato K, Nasuda S, Takumi S. Discovery of highconfidence single nucleotide polymorphisms from large-scale de novo analysis of leaf transcripts of Aegilops tauschii, a wild wheat progenitor. DNA Res. 2012;19:487-97.

15. lehisa JCM, Shimizu A, Sato K, Nishijima R, Sakaguchi K, Matsuda R, Nasuda S, Takumi S. Genome-wide marker development for the wheat D genome based on single nucleotide polymorphisms identified from transcripts in the wild wheat progenitor Aegilops tauschii. Theor Appl Genet. 2014;127:261-71.

16. Nishijima R, Yoshida K, Motoi Y, Sato K, Takumi S. Genome-wide identification of novel genetic markers from RNA sequencing assembly of diverse Aegilops tauschii accessions. Mol Gen Genomics. 2016;291:1681-94.

17. Ramirez-Gonzalez RH, Segovia V, Bird N, Fenwick P, Holdgate S, Berry S, Jack P, Caccamo M, Uauy C. RNA-seq bulked segregant analysis enables the identification of high-resolution genetic markers for breeding in hexaploid wheat. Plant Biotechnol J. 2015;13:613-24.

18. Lu P, Liang Y, Li D, Wang Z, Li W, Wang G, Wang Y, Zhou Q, Xie J, Zhang D, Chen Y, Li M, Zhang Y, Sun Q, Han C, Liu Z. Fine genetic mapping of spot blotch resistance gene Sb3 in wheat (Triticum aestivum). Theor Appl Genet. 2016;129:577-89.

19. International Barley Genome Sequencing Consortium. A physical, genetic and functional sequence assembly of the barley genome. Nature. 2012;491:711-6.

20. Mascher M, Gundlach H, Himmelbach A, Beier S, Twardziok SO, Wicker T, Radchuk V, Dockter C, Hedley PE, Russell J, Bayer M, Ramsay L, Liu H, Haberer G, Zhang X-Q, Zhang Q, Barrero RA, Li L, Taudien S, Groth M, Felder M, Hastie A, Simkova H, Stankova H, Vrana J, Chan S, Munoz-Amatriain M, Ounit R, Wanamaker S, Bolser D, Colmsee C, Schmutzer T, Aliyeva-Schnorr L, Grasso S, Tanskanen J, Chailyan A, Sampath D, Heavens D, Clissold L, Cao S, Chapman B, Dai F, Han Y, Li H, Li X, Lin C, McCoole JK, Tan C, Wang P, Wang S, Yin S, Zhou G, Poland JA, Bellgard MI, Borisjuk L, Houben A, Dolezel J, Ayling S, Lonardi S, Kersey P, Langridge P, Muehlbauer GJ, Clark MD Caccamo M, Schulman AH, Mayer KFX, Platzer M, Close TJ, Scholz U, Hansson M, Zhang G, Braumann I, Spannagl M, Li C, Waugh R, Stein N. A chromosome conformation capture ordered sequence of the barley genome. Nature. 2017;544:427-33.

21. Luo MC, Gu YQ, Puiu D, Wang H, Twardziok SO, Deal KR, Huo N, Zhu T, Wang L, Wang Y, MaGuire PE, Liu S, Long H, Ramasamy RK, Rodriquez JC,
Van SL, Yuan L, Wang Z, Xia Z, Xiao L, Anderson OD, Ouyang S, Liang Y, Zimin AV, Pertea G, Qi P, Bennetzen JL, Dai X, Dawson MW, Müller H-G, Kugler K, Rovarola-Duarte L, Spannagl M, Mayer KFX, Lu F-H, Bevan MW, Leroy P, Li P, You FM, Sun Q, Liu Z, Lyons E, Wicker T, Salzberg SL, Devos KM, Dvorák J. Genome sequence of the progenitor of the wheat D genome Aegilops tauschii. Nature. 2017;551:498-502.

22. Zhao G, Zou C, Li K, Wang K, Li T, Gao L, Zhang X, Wang H, Yang Z, Liu X, Jiang W, Mao L, Kong X, Jiao Y, Jia J. The Aegilops tauschii genome reveals multiple impacts of transposons. Nat Plants. 2017;3:946-55.

23. Mayer KFX, Martis M, Hedley PE, Šimkov H, Liu H, Morris JA, Steuernagel B, Taudien S, Roessner S, Gundlach H, Kubal Kov M, Suchánková P, Murat F, Felder M, Nussbaumer T, Graner A, Salse J, Endo T, Sakai H, Tanaka T, Itoh T, Sato K, Platzer M, Matsumoto T, Scholz U, Doležel J, Waugh R, Stein N. Unlocking the barley genome by chromosomal and comparative genomics. Plant Cell. 2011;23:1249-63.

24. Wicker T, Mayer KFX, Gundlach H, Martis M, Steuernagel B, Scholz U, Šimkov H, Kubal Kov M, Choulet F, Taudien S, Platzer M, Feuillet C, Fahima T, Budak $\mathrm{H}$, Dolezel J, Keller B, Stein N. Frequent gene movement and pseudogene evolution is common to the large and complex genomes of wheat, barley, and their relatives. Plant Cell. 2011;23:1706-18.

25. Bolger AM, Lohse M, Usadel B. Trimmomatic: a flexible trimmer for Illumina sequence data. Bioinformatics. 2014;30:2114-20.

26. Grabherr MG, Haas BJ, Yassour M, Levin JZ, Thompson DA, Amit I, Adiconis X, Fan L, Raychowdhury R, Zeng Q, Chen Z, Mauceli E, Hacohen N, Gnirke A, Rhind N, di Palma F, Birren BW, Nusbaum C, Lindblad-Toh K, Friedman N, Regev A. Full length transcriptome assembly from RNA-seq data without a reference genome. Nat Biotechnol. 2011;29:644-52.

27. Haas BJ, Papanicolaou A, Yassour M, Grabherr M, Blood PD, Bowden J, Couger MB, Eccles D, Li B, Lieber M, Macmanes MD, Ott M, Orvis J, Pochet N, Strozzi F, Weeks N, Westerman R, William T, Dewey CN, Henschel R, Leduc RD, Friedman N, Regev A. De novo transcript sequence reconstruction from RNA-seq using the trinity platform for reference generation and analysis. Nat Protoc. 2013;8:1494-512.

28. Langmead B, Salzberg SL. Fast gapped-read alignment with bowtie 2. Nat Methods. 2012;9:357-9.

29. Li H, Handsaker B, Wysoker A, Fennell T, Ruan J, Homer N, Marth G, Abecasis $G$, Durbin R. Subgroup 1000 genome project data processing. The sequence alignment/map format and SAMtools. Bioinformatics. 2009;25:2078-9.

30. Kosugi S, Natsume S, Yoshida K, MacLean D, Cano L, Kamoun S, Terauchi R. Coval: improving alignment quality and variant calling accuracy for nextgeneration sequencing data. PLoS One. 2013;8:e75402.

31. Kersey PJ, Allen JE, Armean I, Boddu S, Bolt BJ, Carvalho-Silva D, Christensen M, Davis P, Falin LJ, Grabmueller C, Humphrey J, Kerhornou A, Khobova J, Aranganathan NK, Langridge N, Lowy E, McDowall MD, Maheswari U, Nuhn M, Ong CK, Overduin B, Paulini M, Pedro $H$, Perry E, Spudich G, Tapanari E, Walts B, Williams G, Tello-Ruiz M, Stein J, Wei S, Ware D, Bolser DM, Howe KL, Kulesha E, Lawson D, Maslen G, Staines DM. Ensembl genomes 2016: more genomes, more complexity. Nucleic Acids Res. 2015;44:574-80.

32. Wu TD, Watanabe CK. GMAP: a genomic mapping and alignment program for mRNA and EST sequences. Bioinformatics. 2005;21:1859-75.

33. Quinlan AR, Hall IM. BEDTools: a flexible suite of utilities for comparing genomic features. Bioinformatics. 2010;26:841-2.

34. Krzywinski M, Schein J, Birol I, Connors J, Gascoyne R, Horsman D, Jones SJ, Marra MA. Circos: an information aesthetic for comparative genetics. Genome Res. 2009;19:1639-45.

35. Untergasser A, Nijveen $H$, Rao X, Bisseling T, Geurts R, Leunissen JA. Primer3Plus, an enhanced web interface to Primer3. Nucleic Acid Res. 2007; 35:W71-4.

36. Tajima F. Statistical method for testing the neutral mutation hypothesis by DNA polymorphism. Genetics. 1989;123:585-95.

37. Librado P, Rozas J. DnaSP v5: a software for comprehensive analysis of DNA polymorphism data. Bioinformatics. 2009;25:1451-2.

38. Trapnell C, Williams BA, Pertea G, Mortazavi A, Kwan G, van Baren MJ, Salzberg SL, Wold BJ, Pachter L. Transcript assembly and quantification by RNA-seq reveals unannotated transcripts and isoform switching during cell differentiation. Nat Biotechnol. 2010;28:511-5.

39. Matsuoka Y, Takumi S, Kawahara T. Intraspecific lineage divergence and its association with reproductive trait change during species range expansion in central Eurasian wild wheat Aegilops tauschii Coss. (Poaceae). BMC Evol Biol. 2015;15:213. 
40. Fay JC, Wu Cl. Hitchhiking under positive Darwinian selection. Genetics. 2000;155:1405-13.

41. Watterson GA. On the number of segregating sites in genetical models without recombination. Theor Popul Biol. 1975;7:256-76.

42. Jia J, Zhao S, Kong X, Li Y, Zhao G, He W, Appels R, Pfeifer M, Tao Y, Zhang X, Jing R, Zhang C, Ma Y, Gao L, Gao C, Spannagl M, KFX M, Li D, Pan S, Zheng F, Hu Q, Xia X, Li J, Liang Q, Chen J, Wicker T, Gou C, Kuang H, He G, Luo Y, Keller B, Xia Q, Lu P, Wang J, Zou H, Zhang R, Xu J, Gao J, Middleton C, Quan Z, Liu G, Wang J, International wheat genome sequencing consortium, Yang H, Liu X, He Z, Mao L, Wang J. Aegilops tauschii draft genome sequence reveals a gene repertoire for wheat adaptation. Nature. 2013;496:91-5.

43. Luo M-C, Gu YQ, You FM, Deal KR, Ma Y, Hu Y, Huo N, Wang Y, Wang J, Chen S, Jorgensen CM, Zhang Y, MCGuire PE, Pasternak S, Stein JC, Ware D, Kramer M, McCombie WR, Kianian SF, Martis MM, Mayer KFX, Sehgal SK, Li W, Gill BS, Bevan MW, Šimková H, Doležel J, Weining S, Lazo GR, Anderson $\mathrm{OD}$, Dvorak J. A 4-gigabase physical map unlocks the structure and evolution of the complex genome of Aegilops tauschii, the wheat Dgenome progenitor. Proc Natl Acad Sci U S A. 2013;110:7940-5.

44. Zhang H, Jia J, Gale MD, Devos KM. Relationships between the chromosomes of Aegilops umbellulata and wheat. Theor Appl Genet. 1998; 96:69-75.

45. Devos KM, Gale MD. Genome relationships: the grass model in current research. Plant Cell. 2000;12:637-46.

46. Molnár I, Vrána J, Burešová V, Cápal P, Farkas A, Darkó É, Cseh A, Kubaláková M, Molnár-Láng M, Doležel J. Dissecting the U, M, S and C genome of wild relatives of bread wheat (Aegilops spp.) into chromosomes and exploring their synteny with wheat. Plant J. 2016;88:452-67.

47. Nishijima R, Okamoto Y, Hatano H, Takumi S. Quantitative trait locus analysis for spikelet shape-related traits in wild wheat progenitor Aegilops tauschii: implications for intraspecific diversification and subspecies differentiation. PLoS One. 2017;12:e0173210.

48. Sasanuma T, Chabane K, Endo TR, Valkoun J. Characterization of genetic variation in and phylogenetic relationships among diploid Aegilops species by AFLP: incongruity of chloroplast and nuclear data. Theor Appl Genet. 2004;108:612-8.

49. Wright SI, Kalisz S, Slotte T. Evolutionary consequences of self-fertilization in plants. Proc R Soc B. 2013;280:20130133.

Ready to submit your research? Choose BMC and benefit from:

- fast, convenient online submission

- thorough peer review by experienced researchers in your field

- rapid publication on acceptance

- support for research data, including large and complex data types

- gold Open Access which fosters wider collaboration and increased citations

- maximum visibility for your research: over $100 \mathrm{M}$ website views per year

At BMC, research is always in progress.

Learn more biomedcentral.com/submissions 